\section{Community-acquired Pneumonia in Adults}

by PD Dr. med. Martin Kolditz and Prof. Dr. med. Santiago Ewig in issue $49 / 2017$

\section{Adequate Risk Stratification}

Community-acquired pneumonia is one of the main causes of hospital admission, morbidity, and mortality. The case fatality rate of patients who require ICU admission in multicenter cohort studies is more than $40 \%$. The disease course of pneumosepsis is associated with complications and represents a medical emergency, which has to be identified and treated in a timely manner (1).

In clinical practice, diverse scoring models are often used in order to assess patients with regard to the severity of their illness. These models entail various problems, however, which limit their validity. For community-acquired pneumonia (CAP) and CAPassociated sepsis, it is of particular relevance that using different scores can result in different clinical decisions.

The following example illustrates the problem around conventional clinical assessment tools. In a 60-year-old patient with CAP, a respiratory rate of 26 breaths/minute, blood pressure of 95/65 mmHG, blood-urea-nitrogen (BUN) of $18 \mathrm{mg} / \mathrm{dL}$, and a normal mental state, the estimated mortality according to the validated prognostic CURB65 score (developed for pneumonia) is $0.6 \%$, and the patient could therefore receive outpatient treatment. When the qSOFA score (the new scoring tool for patients with suspected sepsis) is applied, however, this patient has likely developed sepsis and should be treated in the intensive care unit $(2,3)$.

This example demonstrates that clinicians need to consider additional parameters to merely applying scoring models. The new omics technologies are likely to enrich our armamentarium in the fight against diseases and enable rapid diagnosis and treatment. DOI: 10.3238/arztebl.2018.0145a

\section{References}

1. Phua J, Dean NC, Guo Q, Kuan WS, Lim HF, Lim TK: Severe community-acquired pneumonia: timely management measures in the first 24 hours. Crit Care 2016; 20: 237

2. Josh Farkas: PulmCrit-top ten problems with the new sepsis definition. www.emcrit. org/pulmcrit/problems-sepsis-3-definition/ (last accessed on 26 February 2017).

3. Singer M, Deutschman CS, Seymour CW, et al.: The third international consensus definitions for sepsis and septic shock (Sepsis-3). JAMA 2016; 315: 801-10.

4. Kolditz M, Ewig S: Community-acquired pneumonia in adults. Dtsch Arztebl Int 2017; 114: 838-48.

\section{Dr. med. Nikolaos Evangelatos}

Medizinische Klinik 3, Internistische Intensivstation, Klinikum Nürnberg

Nikolaos.Evangelatos@klinikum-nuernberg.de

Dr. med. Thomas Gerlach

Medizinische Klinik 6, Zentrale Notaufnahme, Klinikum Nürnberg

Thomas.Gerlach@klinikum-nuernberg.de

\section{Conflict of interest statement}

The authors declare that no conflict of interest exists.
In Reply:

We thank Evangelatos and Gerlach for their contribution, in which they emphasize again the importance of adequate risk stratification, aiming to identify patients with acute organ dysfunction in pneumonia early on. The case fatality rate they mention - of $40 \%$ in inpatients - however, relates to patients with severe pneumonia in intensive care wards, whereas, according to the data of a German nationwide external quality program, inpatients in Germany with CAP still have a case fatality rate of $13 \%$ (1).

The example case mentioned by Evangelatos and Gerlach illustrates the problems with arbitrary thresholds of parameters used in scores. It clarifies once again that the clinical assessment of an experienced doctor remains a central element of risk stratification, which should be objectified by applying validated parameters and follow-up assessments.

The qSOFA score was developed as a screening instrument for sepsis and should as such not trigger direct admission to intensive care but extended diagnostic evaluation with regard to acute organ dysfunction (for example, by using the SOFA score or minor criteria). The higher sensitivity of qSOFA compared with the $\mathrm{C}(\mathrm{U}) \mathrm{RB}-65$ score that the authors described in their contribution has recently been confirmed in the German CAPNETZ cohort in pneumonia, but comes at the cost of a lower specificity (2). The $\mathrm{C}(\mathrm{U}) \mathrm{RB}-65$ score is much better validated in pneumonia.

Developments in omics technologies to optimize risk stratification, with the aim of implementing individualized therapeutic algorithms, are promising new approaches, also in severe pneumonia (3), but these are currently not available in clinical practice settings. In Germany, the multicenter PROGRES-CAP Study is investigating this research question in pneumonia; initial results are expected in 2018 .

DOI: $10.3238 / a r z t e b l .2018 .0145 b$

\section{References}

1. IQTIG - Institut für Qualitätssicherung und Transparenz im Gesundheitswesen: Bundesauswertung zum Erfassungsjahr 2016: Ambulant erworbene Pneumonie. https://iqtig.org (last accessed on 31 January 2018).

2. Kolditz M, Scherag A, Rohde G, Ewig S, Welte T, Pletz M, CAPNETZ Study Group: Comparison of the qSOFA and CRB-65 for risk prediction in patients with communityacquired pneumonia. Intensive Care Med 2016; 42: 2108-10

3. Davenport EE, Burnham KL, Radhakrishnan J, et al.: Genomic landscape of the individual host response and outcomes in sepsis: a prospective cohort study. Lancet Respir Med 2016; 4: 259-71.

4. Kolditz M, Ewig S: Community-acquired pneumonia in adults. Dtsch Arztebl Int 2017; 114: 838-48.

\section{PD Dr. med. Martin Kolditz}

Abteilung für Pneumologie

Medizinische Klinik und Poliklinik I

Universitätsklinikum Carl Gustav Carus

Dresden

martin.kolditz@uniklinikum-dresden.de

\section{Conflict of interest statement}

PD Dr. Kolditz has served as a paid consultant for Astra-Zeneca, Bayer, and Basilea. $\mathrm{He}$ has received third-party funding for a research project from Pfizer, reimbursement of travel expenses from Pfizer und Astra-Zeneca, and lecture honoraria from Pfizer, AstraZeneca, Bayer and Roche. 